Service social

\title{
Comment faciliter la réussite de nos interventions malgré un contexte difficile
}

\section{Dave Ward}

Volume 46, numéro 2-3, 1997

Groupes - Symposium 1997

URI : https://id.erudit.org/iderudit/706759ar

DOI : https://doi.org/10.7202/706759ar

Aller au sommaire du numéro

Éditeur(s)

École de service social de l'Université Laval

ISSN

1708-1734 (numérique)

Découvrir la revue

Citer cet article

Ward, D. (1997). Comment faciliter la réussite de nos interventions malgré un contexte difficile. Service social, 46(2-3), 57-64. https://doi.org/10.7202/706759ar d'utilisation que vous pouvez consulter en ligne.

https://apropos.erudit.org/fr/usagers/politique-dutilisation/ 


\section{Comment faciliter la réussite de nos interventions malgré un contexte difficile}

Dave WARD

Sous le thème «Par-delà les frontières » nous ferons un survol du travail de groupe en Grande-Bretagne en nous attardant aux solutions qui pourraient être les plus appropriées. Nous demandons aux lecteurs d'Amérique du Nord de faire le lien avec leur propre pratique suivant le contexte organisationnel et culturel qui est le leur.

D'entrée de jeu, je vous invite à partager le souvenir d'une expérience personnelle qui me força à m'interroger sur la nature et la place du travail social de groupe. Au début de 1997, on me demanda d'écrire un chapitre sur le travail de groupe pour un nouveau manuel destiné aux étudiants de travail social (Adams et collab., 1998). Après avoir d'abord cru que la tâche serait assez simple, je me heurtai à une difficulté de taille. Quand je m'intéressai aux lieux de travail où la plupart des lecteurs du manuel feraient l'apprentissage de leur champ de pratique et où ils travailleraient vraisemblablement, je constatai en effet que le travail de groupe était devenu pour ainsi dire inexistant. Qui plus est, cette absence se confirma lorsque je fouillai la documentation professionnelle produite en Grande-Bretagne dans les dernières années. Après une abondance d'écrits sur le sujet dans les années 1970 et 1980, la production s'est appauvrie et, quand elle existe, elle se situe aujourd'hui en marge de la pratique du service social. 
Il est nécessaire ici de dire un mot sur l'organisation du travail social en Grande-Bretagne. Dans ce pays, le travail social est dominé par ce qu'on appelle les « responsabilités statutaires ». II s'agit d'obligations légales imposées surtout aux autorités municipales en matière de protection des enfants, de soutien aux handicapés mentaux et aux délinquants, de services pratiques aux personnes âgées et aux handicapés, tâches généralement remplies par les travailleurs sociaux. Historiquement, la plus grande partie de ce travail a été assumée par les départements municipaux. Plus récemment, cependant, la mainmise des agences gouvernementales a été minée par un test de marché des services d'assistance sociale et par la distinction qui s'est établie entre les fonctions d'acheteur et de fournisseur, amenant une expansion des secteurs "volontaires " et commerciaux des services sociaux. Le travail auprès des individus est prépondérant, mais on ne l'appelle pas " casework " en raison de l'association de ce terme avec les approches psychodynamiques. De tout temps l'organisation communautaire s'est trouvée à l'extérieur du courant principal des services sociaux, donc, du travail social, et le travail de groupe a été vu comme un ajout facultatif au travail avec les individus. C'est ainsi que le travail de groupe a toujours été marginal, bien qu'il ait connu une période de " gloire" dans les années 1970 et 1980. D'ailleurs, les énormes compressions budgétaires des années 1990 ont recentré la pratique sur les obligations légales et, en même temps, sur une approche individualisée.

Mais revenons à mon anecdote. Je conclus alors rapidement que je ne pouvais pas, en toute conscience, donner un aperçu sommaire de la théorie sous-tendant le travail de groupe, pas plus que des indications sur sa pratique. Si je le faisais, je perdrais tout contact avec la réalité et donnerais prise aux critiques fréquentes des étudiants, qui ne manqueraient pas de trouver que les textes qu'on leur fournit ne collent pas à leur nouvelle réalité professionnelle. Je me tournai donc plutôt, comme je le fais chaque fois qu'une difficulté se présente, vers la méthode du travail de groupe autodirigé (Mullender et Ward, 1991) pour aborder les problèmes. Je décidai de rédiger un texte basé sur les questions suivantes:

QU'EST-CE QUI est arrivé au travail de groupe?

POURQUOI?

COMMENT pouvons-nous faire quelque chose à ce sujet? 


\section{Qu'est-il arrivé?}

\begin{tabular}{lll}
\hline $\begin{array}{l}\text { Le travail de groupe } \\
\begin{array}{l}\text { Le groupe comme } \\
\text { instrument de } \\
\text { changement }\end{array}\end{array}$ & $\begin{array}{l}\text { est devenu } \\
\text { est devenu }\end{array}$ & $\begin{array}{l}\text { le travail en groupe } \\
\text { le groupe comme } \\
\text { contexte d'intervention }\end{array}$ \\
$\begin{array}{l}\text { Le souci d'une } \\
\text { dynamique de rencontre } \\
\text { de groupe }\end{array}$ & est devenu & $\begin{array}{l}\text { une orientation } \\
\text { pédagogique fondée } \\
\text { sur les instructions }\end{array}$ \\
$\begin{array}{l}\text { Les valeurs } \\
\text { démocratiques } \\
\text { et collectives }\end{array}$ & sont devenues & $\begin{array}{l}\text { des valeurs autoritaires } \\
\text { et individualistes }\end{array}$ \\
\hline
\end{tabular}

Au cours des dernières années, on a perçu une tendance vers des groupes de plus en plus orientés vers la tâche avec toujours moins d'accent mis sur la méthode, réduisant ainsi le travail de groupe à un exercice stérile à l'intérieur duquel les membres reçoivent des programmes globaux qui ne les reconnaissent pas comme des personnes uniques, souvent étouffées par les conditions sociales de pauvreté qui sont les leurs. On ne s'intéresse pas - sinon très peu à la dynamique de groupe, pas plus qu'aux habiletés et aux méthodes du travail de groupe.

On observe particulièrement ces caractéristiques dans les programmes cognitifs béhavioraux basés sur le groupe, actuellement fort répandus en Grande-Bretagne, reflet de ce que Konopka (1970) décrit comme un traitement en face à face où les autres participants font office de spectateurs (cité par Kurland et Salmon, 1993, p. 8). Écrivant au sujet du travail auprès d'abuseurs sexuels mâles, Cowburn et Modi (1995), deux praticiens anglais, voient dans de telles approches des conceptions eurocentriques et hétérosexistes, basées sur la conformité et l'obéissance, mais dans les faits potentiellement dangereuses en ce qu'elles confortent les abuseurs dans leurs vues minimales de leur propre responsabilité et du tort que leurs gestes ont causé.

Ces changements peuvent être replacés à l'intérieur d'un mouvement sociopolitique et économique plus large qui a influé sur le travail social durant les années 1980 et 1990 . Un nouveau climat idéologique où il est loisible à un premier ministre britannique d'affirmer 
que « la société n'existe pas », l'ouverture aux pratiques commerciales pour la fourniture des services sociaux, une nouvelle vision " managériale », une tendance vers les spécialités client-usager, le recours à la loi comme facteur clé de définition, de nouvelles façons de voir l'enseignement et l'apprentissage du travail social, fondées sur la compétence... tous ces éléments font en sorte qu'on a délaissé plusieurs des valeurs et des objectifs du travail de groupe. Ces derniers impliquent que :

- le processus central du travail de groupe consiste en l'interaction entre un groupe de personnes fondée sur la réciprocité, comme moyen d'atteindre un but de groupe;

- dans son contexte, son but, sa méthode, ainsi que les relations et les comportements manifestés dans les rencontres, le travail de groupe se situe à l'encontre de l'oppression (Brown, 1996, p. 83).

Le travail de groupe peut avoir été délaissé justement parce qu'il reconnaît que le groupe possède une vie qui lui est propre, que l'intervenant ne peut jamais contrôler complètement. Dans un groupe, l'ordre du jour est normalement une vue de l'ensemble et le processus est démocratique. Les membres du groupe vont faire valoir ce qui est important pour eux, peu importe les règles établies. De telles caractéristiques détonnent dans le courant actuel où l'accent est mis sur la discipline et sur la responsabilité individuelle et, au niveau de l'organisation, sur des objectifs prédéterminés et des résultats attendus. La conséquence est que plusieurs projets et travailleurs ignorent totalement l'idée d'un véritable travail de groupe ou, pire, s'en méfient.

\section{Comment devons-nous réagir?}

Je crois que la tradition anglo-américaine nous cause un problème. Fondée sur la compétition et l'individualité, elle entre en conflit avec nos propres croyances. Cependant, Brown (1996) relève trois éléments clés pour l'avenir du travail de groupe : les valeurs, la construction d'un modèle basé sur la pratique ainsi que le soutien au travail de groupe et aux intervenants de groupe en cette fin de millénaire (p. 90). Ce que j'aimerais proposer maintenant est une réaffirmation pratique du travail de groupe qui puisse couvrir ces considérations. 
Dans une communication récente, le National Institute for Social Work in Britain (1996) fait ressortir que la pierre angulaire de la politique sociale, et un défi pour le travail social dans cette nouvelle Europe qui s'agrandit, est la lutte contre l'exclusion sociale. II maintient que le défi de la réduction de l'exclusion sociale appelle de nouvelles façons de voir l'accès des personnes et des groupes à l'emploi et aux services, accès qui leur est actuellement refusé.

Dans ce contexte il nous faut retourner au lien historique entre le travail de groupe, d'une part, et l'égalité et la démocratie, d'autre part. À l'occasion de mon travail avec des collègues d'Europe, de l'Est et de l'Ouest, je suis stimulé quand j'observe, dans d'autres pays et cultures où le chômage de masse, les changements démographiques et l'impuissance apparente des gouvernements à fournir une assistance sociale ont engendré diverses formes d'exclusion sociale, que des efforts conscients sont faits pour donner aux services sociaux un nouveau cadre qui met l'accent sur une approche d'inclusion orientée vers le groupe. À l'opposé, au RoyaumeUni, nous avons à peine commencé à nous pencher sur le rôle des travailleurs sociaux comme agents de lutte contre l'exclusion sociale et sur l'élaboration de modèles de pratique visant une perspective plus large.

Le défi constitué par l'exclusion sociale demande que nous tenions compte de ce que nous avons appris sur l'oppression systématique des groupes exclus, sur les pratiques antidiscriminatoires et sur l'empowerment. Dans ce contexte, les fondements du travail de groupe montrent bien toute leur importance. La raison en est que le groupe peut être :

- une source de soutien immédiat, d'amitié;

- un endroit pour reconnaître les expériences partagées et leur valeur;

- une façon de vaincre l'isolement et la solitude;

- la source d'une perspective différente sur les problèmes personnels;

- un endroit où exercer son pouvoir sur des situations personnelles avec la capacité de les changer et d'agir sur elles (Butler et Wintram, 1991, p. 77). 
Au-delà de ces motifs, « il est plus facile d'aborder dans le groupe les difficultés d'ordre privé et les questions d'ordre public comme entités inséparables " (Breton, 1994, p. 31). Bien que des préoccupations puissent apparaître comme des ennuis personnels, dans un groupe ces ennuis personnels deviennent bientôt des problèmes partagés qui fournissent matière à analyse de leurs causes structurelles et à action collective pour susciter le changement (Mullender et Ward, 1991).

Afin d'illustrer ce qui est possible quand les fondements sont en place, je conclurai par des exemples de travail de groupe tirés de ce qu'on peut considérer comme les secteurs les moins prometteurs de la pratique du service social.

Sandra Butler (1994) écrit sur un groupe destiné à des femmes dont les enfants sont considérés par les services sociaux comme courant de grands risques d'être maltraités par leurs parents. Elle observe comment le style " facilitant » du travail de groupe a créé un climat d'égalité, ce qui a rendu ces femmes capables d'explorer l'humour, la tristesse et les contraintes de la vie de famille, au lieu de se taire à ce sujet (p. 178). Tenant une place centrale dans l'agenda du groupe, les problèmes structurels devant lesquels les membres se trouvaient ont émergé : la sexualité et les rapports des femmes avec leur partenaire, mêlés à un processus de racisme et aux difficultés d'élever des enfants nés de parents de races différentes. Devant le sort pénible qui était le leur, ces femmes ont facilement pointé les politiques liées à la pauvreté. Mettre en évidence les problèmes structurels et individuels a été déterminant pour la prise en main de ces femmes et pour la création de leurs propres solutions aux menaces ou aux dilemmes qu'elles devaient affronter (Butler, 1994, p. 163).

Dans le contexte complètement différent d'un pénitencier pour jeunes contrevenants, Bill Badham et ses collaborateurs (1989) ont travaillé avec les pensionnaires de l'établissement pour mettre sur pied un service intégré de transition. Ces jeunes hommes ont trouvé le service utile et approprié en ce qu'il les a rendus aptes à soulever des questions et des plaintes à l'intérieur de l'établissement et les a préparés à la sortie. Les travailleurs sociaux ont cherché à faire sortir de ces jeunes hommes - et à leur fournir les moyens d'acquérirles habiletés et les connaissances qui les aideraient à survivre à la 
fois « dedans " et après leur libération. Pour faire face aux attitudes racistes et sexistes faisant partie de cet apprentissage, l'équipe d'intervenants comprenait des femmes et des Noirs. Les jeunes hommes décidaient du programme du groupe, produisant, à la suite de vastes discussions, des plaquettes d'information et organisant des conférences sur les droits à la sécurité du revenu, le logement, la libération conditionnelle et la liberté provisoire. En ce qui concerne les conférenciers invités, y compris le gouverneur, le groupe a fait les préparatifs pour les allocutions, s'est chargé de faire les invitations et a formulé des questions afin d'obtenir l'information souhaitée. Le gouverneur a reconnu la valeur du forum et a vu dans ce contexte différent les capacités jusque-là ignorées de ces pensionnaires. Un jeune homme a dit en quittant le groupe :

On avait l'impression qu'on pouvait vraiment faire quelque chose de notre vie pendant qu'on était en dedans (Badham, 1989, p. 35).

On a bien là un modèle de pratique en travail de groupe visant à contrer le processus d'exclusion. Cette forme de travail de groupe qui est fondamentalement démocratique favorise la prise en charge; elle a une place au cœur de la démocratie et de l'empowerment. Évidemment, nous ne devons pas être complaisants ni manquer d'esprit critique, mais, à l'intérieur du travail de groupe, il y a des valeurs sûres dont nous pouvons être fiers et que nous devons nous efforcer de retrouver et de posséder à nouveau.

Dave WARD

Professeur Department of Social and Community Studies

De Montfort University

Leicester, Angleterre

\section{Références bibliographiques}

Adams, R., L. Dominelli et M. PAYNe (dir.) (1998). Social Work : Themes, Issues and Critical Debates, Londres, Macmillan.

BADHAM, B. (dir.) (1989). " Doing Something with our Lives when We're Inside: Self-Directed Groupwork in a Youth Custody Centre ", Groupwork, vol. 2, n’ 1, p. 27-35. 
BRETON, M. (1994). « On the Meaning of Empowerment and Empowerment. Oriented Social Work Practice ", Social Work with Groups, vol. 17, $n^{\circ}$ 3, p. 23-38.

Brown, A. (1996). "Groupwork into the Future: Some Personal Reflections ", Groupwork, vol. 9, n 1, p. 80-96.

BUTLER, S. (1994). " "All I've got in my purse is mothballs!", The Social Action Women's Group ", Groupwork, vol. 7 n², p. 163-179.

BUTLER, S. et C. WINTRAM (1991). Feminist Groupwork, Londres, Sage.

COWBURN, M. et P. MODI (1995). " Justice in an Unjust Context : Implications for working with adult male sex offenders ", dans D. Ward et $M$. Lacey (dir.), Probation : Working for Justice, Londres, Whiting and Birch.

KONOPKA, G. (1990). "Thirty-Five Years of Group Work in Psychiatric Settings ", Social Work With Groups, vol. 13, n 1, p. 13-16.

KURLAND, R. et R. SALMON (1993). " Groupwork versus Casework in a Group ", Groupwork, vol. 6, n 1, p. 5-16.

MULLENDER, A. et D. WARD (1991). Self-Directed Groupwork : Users Take Action for Empowerment, Londres, Whiting and Birch.

NATIONAL INSTITUTE FOR SOCIAL WORK (1996). Social Exclusion. Civil Society and Social Work. Briefing No. 18, Londres, NISW. 